Disponível em

http://www.anpad.org.br/rac

RAC, Curitiba, v. 15, n. 4, art. 5, pp. 650-669, Jul./Ago. 2011

(c) EY-NG

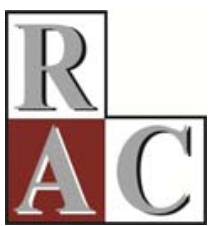

\title{
Gestão de Recursos Humanos: uma Metanálise de seus Efeitos sobre Desempenho Organizacional
}

\author{
Human Resource Management: a Meta-analysis of its Effects on Organizational \\ Performance
}

\begin{abstract}
Agostinha Mafalda Barra de Oliveira *
E-mail: agostinhamafalda@ufersa.edu.br Universidade Federal Rural do Semi-Árido - UFERSA Mossoró, RN, Brasil.

Alexandre José de Oliveira E-mail: alexandreoliveira@ufersa.edu.br Universidade Federal Rural do Semi-Árido - UFERSA Mossoró, RN, Brasil.
\end{abstract}

* Endereço: Agostinha Mafalda Barra de Oliveira

Av. Francisco Mota, 572, Bairro Costa e Silva, Mossoró/RN, 59625-900.

Copyright (C) 2011 RAC. Todos os direitos, até mesmo de tradução, são reservados. É permitido citar parte de artigos sem autorização prévia, desde que seja identificada a fonte. 


\title{
Resumo
}

O campo de estudo da Gestão Estratégica de Recursos Humanos e, mais especificamente, da relação entre práticas de Gestão de Recursos Humanos (GRH) e desempenho organizacional, apresenta controvérsias que ainda precisam ser resolvidas. Isto significa que permanece a necessidade de revisar a literatura dos estudos neste campo, para encontrar algum sentido no vasto número de conclusões propostas. Por esse motivo, a fim de atualizar e acatar sugestões e examinar moderadores, ainda não analisados em outras revisões, a proposta para o presente estudo é realizar uma metanálise dos resultados de 194 registros de estudos empíricos e originais, realizados e/ou publicados entre os anos de 1985 a 2007, mantendo a metanálise por uma ou mais práticas de GRH e por um ou mais indicadores de desempenho organizacional. Para a realização desta metanálise escolhemos o método de metanálise psicométrica de Hunter e Schmidt (2004). Com base nesta metanálise, podemos inferir que há uma relação positiva entre GRH e desempenho organizacional. A estimação da magnitude desta relação aumenta, quando as práticas de GRH são combinadas em um sistema ou dois ou mais resultados de desempenho organizacional são levados em consideração.

Palavras-chave: gestão estratégica de recursos humanos; desempenho organizacional; metanálise.

\begin{abstract}
The field of Strategic Human Resource Management, especially the relationship between Human Resource Management (HRM) practices and organizational performance, has controversies that remain unresolved. This means that there is still a need to review the literature of this field in order to find some meaning in the large number of proposed conclusions. Therefore, in order to update, accept suggestions and examine moderators not analyzed in other reviews, this study aims to conduct a meta-analysis of the results of 194 registers of empirical and original studies, conducted and/or published from 1985 to 2007, concerning one or more practices of HRM and one or more organizational performance indicators. For the meta-analysis, Hunter and Schmidt's (2004) psychometric meta-analysis method was chosen. From this meta-analysis it is possible to conclude that there is a positive relationship between HRM and organizational performance. Estimation of the magnitude of this relationship increases when the HRM practices are combined into one system or two or more organizational performance outcomes are considered.
\end{abstract}

Key words: strategic human resource management; organizational performance; meta-analysis. 


\section{Introdução}

A revisão realizada por Ferris, Hochwarter, Buckley, Harrell-Cook e Frink (1999) sobre a teoria, pesquisa e prática em GRH indicou que a GRH tem evoluído, em grande medida, de uma função de manutenção, com pouco impacto financeiro, para uma fonte da vantagem competitiva sutentável das organizações. Nesse sentido, Becker e Huselid (1998a) explicaram que a evolução da GRH, para o nível estratégico, deu-se, principalmente, pelo reconhecimento de que uma força de trabalho qualificada, motivada e alinhada com as necessidades da organização pode prover a organização de um ativo necessário nos processos organizacionais requeridos pelos novos imperativos de mercado.

Nessa mesma linha de raciocínio, Lacombe (2004) concluiu que o progresso no campo de pesquisa da GRH deu-se, em parte, devido à preocupação da área em agregar valor à organização. Adicionalmente, Wright, Gardner, Moynihan e Allen (2005) afirmaram que o interesse em pesquisas sobre este tema veio como resposta ao longo processo histórico de desvalorização, enfrentado pela área de GRH como geradora de custos e não agregadora de valor.

Dessa maneira, muitos estudos têm sido realizados, a fim de verificar a relação existente entre práticas de GRH e desempenho organizacional. Contudo, as revisões realizadas sobre estes estudos apontaram divergências tanto teóricas quanto metodológicas.

As revisões qualitativas descreveram, ou sugeriram, que há uma relação positiva entre práticas de GRH e desempenho organizacional (Lacombe, 2004; Ulrich, 1997; Wood, 1999a; Wright, Gardner, Moynihan, \& Allen, 2005). Ou, mais especificamente, entre práticas de trabalho de alto desempenho (PTAD) e desempenho organizacional (Guest, 1997; Ichniowski, Kochan, Levine, Olson, \& Strauss, 1996). No entanto essas revisões concluíram que os estudos empíricos avaliados não explicaram de que maneira esta relação se processava (Guest, 1997; Lacombe, 2004; Wright et al., 2005).

De outra forma, as revisões quantitativas (Combs, Liu, Hall, \& Ketchen, 2006; Guzzo, Jette, \& Katzell, 1985; Oliveira, 2006; Oliveira, González, \& Oliveira, 2007a, 2007b) encontraram evidências para comprovar a existência de um relacionamento significativo e positivo entre GRH e desempenho organizacional. Ademais, as práticas de GRH, quando avaliadas em conjunto, obtiveram melhores estimadores pontuais do que quando avaliadas em separado. Entretanto não há acordo quanto à identificação dessas práticas, quando analisadas isoladamente.

Em síntese, o campo de estudo da Gestão Estratégica de Recursos Humanos (GERH) e, mais especificamente, da relação entre práticas de GRH e desempenho organizacional, apresenta controvérsias que ainda precisam ser resolvidas. Isto significa que permanece a necessidade de se revisar a literatura dos estudos neste campo, para encontrar algum sentido no vasto número de conclusões propostas. Pelo exposto, a fim de atualizar, acatar sugestões e examinar moderadores ainda não analisados nas revisões apresentadas, a proposta para o presente estudo é de utilizar a metodologia metanalítica e investigar os resultados dos estudos empíricos que avaliaram a relação entre GRH e desempenho organizacional.

\section{Gestão de Recursos Humanos}

A operacionalização da GRH, em nível operacional ou estratégico, é alcançada por meio de práticas pertinentes ao gerenciamento de pessoas nas organizações. São muitas as práticas destinadas para este gerenciamento. O domínio, a extensão e a formalidade dada a cada uma das práticas de GRH dependem das contingências internas e externas da organização. 
Cada vez mais atenção tem sido devotada à integração que deve existir entre as práticas de GRH (ajuste interno), entre estas práticas e as contingências internas e externas à organização (ajuste externo), ou mais especialmente, entre as práticas de GRH e a estratégia da organização (Barrette \& Ouellette, 2000; Becker \& Gerhart, 1996; Paauwe \& Boselie, 2005; Schuler \& Jackson, 1987; Wood, 1999a). Para Becker e Huselid (1998b) e, mais recentemente, para Arthur e Boyles (2007), as complementaridades ou sinergias desses elementos são dimensões importantes para entender a relação entre GRH e desempenho organizacional.

\section{Sistemas de práticas de gestão de recursos humanos}

No intuito de alcançar o ajuste interno e externo, muitos estudiosos defendem a existência de um sistema ou de sistemas de práticas de GRH especiais ou superiores (Guthrie, 2001; Macky \& Boxall, 2007; Pfeffer, 1998; Sanz-Valle \& Sánchez, 2000). Segundo Way (2002), os sistemas de práticas de GRH especiais diferem dos sistemas de práticas de GRH comuns, por possuírem habilidades superiores e aplicarem estas atividades em questões laborais que resultarão em melhor desempenho organizacional. Em complemento, Combs, Liu, Hall e Ketchen (2006), explicaram que os sistemas de práticas de GRH especiais impactam positivamente o desempenho organizacional pelo fato de desenvolverem as competências dos empregados; dar autonomia e oportunidade; influenciar e motivar estes empregados para utilizar suas competências em benefício da organização e, principalmente, atuar ao longo das estruturas sociais internas de uma organização.

Estes sistemas têm recebido diferentes denominações. A denominação mais destacada é a de práticas de alto desempenho (Den Hartog \& Verburg, 2004; Huselid, 1995; Huselid \& Becker, 1995; Way, 2002; Zacharatos, Barling, \& Iverson, 2005). Ou, de maneira alternativa, de práticas de alto compromisso (Burton \& O'Reilly, 2000; Chang, 2005; Collins \& Smith, 2006; Gould-Williams, 2004). Há também de práticas de alto envolvimento (Guerrero \& Barraud-Didier, 2004; Guthrie, Spell, \& Nyamori, 2002; Macky \& Boxall, 2008); ou práticas de construção do capital humano (Audea, Teo, \& Crawford, 2005; Zhu, Chew, \& Spangler, 2005). Ademais de práticas progressivas, alternativas ou inovadoras (Agarwala, 2003; Delaney \& Huselid, 1996; Ichniowski \& Shaw, 1999), entre outras denominações.

No entanto todas estas denominações, de alguma forma, representam um tipo de gerenciamento integrado mais adequado às condições atuais e competitivas que permeiam as organizações. Pelo exposto, os sistemas de GRH especiais; ou a partir desse momento Sistemas de Práticas de GRH (SPGRH), são unidades de trabalho integradas que, sustentavelmente, podem agregar valor organizacional, devido aos seus processos e estruturas únicas. A natureza e características destes SPGRH os convertem em ativos estratégicos que podem aportar uma vantagem competitiva para a organização adicionando valor e mantendo-a em tempo, por tratar-se de ativos que não se depreciam, são difíceis de imitar, substituir ou transferir.

A descoberta, a síntese e a descrição dos resultados de pesquisas existentes já apoiam a suposição de que os SPGRH apresentam efeitos mais significativos do desempenho organizacional do que quando as práticas de GRH são analisadas separadamente (Combs et al., 2006; Oliveira, 2006). Isto significa que, quando as práticas são desenhadas e implantadas em sinergia, o efeito coletivo destas práticas poderá ser maior do que a soma de suas partes individuais (Wall \& Wood, 2005; Wood, 1999a, 1999b). Esta suposição corrobora o argumento de Barney (1995): as práticas de GRH, quando em combinação com outros recursos e capacidades, podem capacitar uma organização para alcançar plenamente alcançar sua vantagem competitiva; portanto estabelecemos como nossa primeira hipótese.

H1: O tamanho do efeito estimado para SPGRH e desempenho organizacional será maior do que o tamanho do efeito estimado para cada prática de GRH em separado e desempenho organizacional. 


\section{Desempenho Organizacional}

Com base no modelo expandido de desempenho organizacional, proposto por Rogers e Wright (1998), Way e Johnson (2005) defenderam que a medida de desempenho organizacional para a GRH deve ser concebida como construto multidimensional. De acordo com Colakoglu, Lepak e Hong (2006), como construto multidimensional, desempenho organizacional em GRH varia pelo quão próximo ele é para o planejado impacto de práticas de GRH, o nível ao qual ele está agregado, e pelos grupos de stakeholders cujos interesses são atendidos; portanto os desenhos de pesquisa em GRH que incorporam uma simples ou estreita perspectiva de desempenho organizacional, provavelmente, não oferecem possibilidades para apuradamente explicar ou avaliar a principal relação entre GRH e desempenho organizacional.

Em suma, nas palavras de Ferris et al. (1998), desempenho organizacional é necessariamente um construto multidimensional, mais político do que científico. Múltiplos critérios de efetividade são necessários para considerar os diversos elementos internos e externos pelos quais as organizações são responsáveis. Critérios de efetividade potencialmente incluem resultados de recursos humanos, resultados organizacionais, resultados financeiros e contábeis, e resultados de mercado de capital. Esta definição de efetividade organizacional amplia o conceito de desempenho organizacional e inclui a tipologia de outcomes desenvolvida por Dyer e Reeves (1995).

\section{Tipologia de outcomes de Dyer e Reeves}

A escolha dos indicadores para medir desempenho organizacional é uma questão importante para avaliar o impacto da GRH sobre esse desempenho. Com este foco, Dyer (1984) apresentou algumas possibilidades de outcomes ou resultados provenientes das ações de GRH. A classificação destas possibilidades, proposta por Dyer (1984) e, posteriormente expandida por Dyer e Reeves (1995), envolve quatro tipos de resultados possíveis, que são denominados resultados de recursos humanos, resultados organizacionais, resultados financeiros e resultados de mercado capital.

Os resultados de recursos humanos constituem resultados ou consequências de GRH sobre as competências dos empregados individualmente. Por sua vez, os resultados organizacionais constituem resultados de GRH sobre o conjunto de competências dos empregados transformados em competências organizacionais. Os resultados financeiros ou contábeis são as consequências das práticas sobre índices financeiros. Por fim, os resultados de mercado de capital são as consequências das práticas de GRH sobre a valorização da organização no mercado financeiro.

De acordo com Dyer (1984), os quatro outcomes, de recursos humanos, organizacionais, financeiros e de mercado de capital devem ser avaliados em conjunto. Somente uma avaliação conjunta desses outcomes torna possível inferir conclusões mais plausíveis sobre a natureza da relação entre GRH e desempenho organizacional, bem como de suas próprias inter-relações, moderações e hierarquias.

\section{Cadeia sequencial de outcomes}

Em relação ao impacto de GRH sobre estes resultados, Dyer e Reeves (1995) argumentaram que há uma cadeia sequencial dos outcomes apresentados. Duas suposições podem ser extraídas deste argumento: o fato de que as estratégias de GRH são principalmente desenhadas para impactar outcomes de recursos humanos, e que o aumento da complexidade de fatores que influenciam altos níveis de outcomes poderia diminuir a relativa contribuição de fatores de GRH para aqueles outcomes. Estas duas suposições e mais a constatação da realidade de que outcomes de recursos humanos são deficientes sob o ponto de vista de muitos executivos explicam por que muitas das pesquisas de GERH focalizam mais outcomes organizacionais do que os outros três tipos de outcomes. 
Por sua vez, Guest (1997), avaliando a relação entre GRH e desempenho organizacional, questionou a distância causal entre um input de GRH e os outputs baseados no desempenho financeiro, pois são muitos os fatores que podem afetar esta relação. Nesse sentido, o uso de indicadores de outcomes mais próximos, particularmente aqueles em que as ações de GRH têm influencia mais direta, é mais plausível e metodologicamente mais fácil para fazer correlações. Por esse motivo, Guest (1997) desenvolveu um modelo que explica o impacto do processo de GRH sobre o desempenho organizacional.

Depois de Guest (1997), outros estudiosos, tais como Liouville e Bayad (1998), Koys (2001), Paul e Anantharaman (2003) e Guthrie, Datta e Wright (2004) também desenvolveram seus próprios modelos. O argumento de Dyer e Reeves (1995) e os diversos modelos propostos têm ganhado atenção na literatura de GERH. Em comum, todos eles defendem que outcomes de recursos humanos, como também outcomes organizacionais, podem servir de moderadores entre GRH e outcomes financeiros e de mercado.

Em outras palavras, os outcomes podem ser diferenciados sobre níveis hierárquicos, com outcomes sobre um nível, contribuindo, junto com outros fatores, para outcomes dos níveis mais próximos. Assim, embora haja uma variação quanto ao número de níveis e quanto à definição exata de outcomes, todos estes modelos, de forma genérica, descrevem as práticas de GRH como tendo seu mais direto impacto sobre outcomes de recursos humanos que, em retorno, contribui para níveis mais altos de construtos de desempenho organizacional (Rogers \& Wright, 1998). De maneira geral, a literatura de GRH vem acumulando evidências de que os outcomes de recursos humanos e organizacionais podem servir como mediadores na relação entre GRH e desempenho organizacional.

No entanto, Combs et al. (2006) investigaram esse efeito mediador, em uma metanálise, com alguns dos indicadores de desempenho de cada resultado; verificaram que as medidas de turnover (resultado de recursos humanos) e de produtividade (resultado organizacional) não apresentaram tamanhos de efeito maiores do que as medidas de retorno contábil e crescimento (resultados financeiros) e retorno de mercado (resultado de mercado). Isto significa que o grau de magnitude do impacto de GRH sobre o desempenho organizacional foi independente dos indicadores de outcomes utilizados. A explicação mais plausível que esses autores encontraram para essa constatação foi que, diferente de outras funções, a função de gerenciar pessoas permeia toda a organização e, por conseguinte, afeta de maneira complexa e simultânea todos os seus resultados, sejam recursos humanos, organizacionais, financeiros ou de mercado.

Way e Johnson (2005) ofereceram uma explicação detalhada do impacto direto de GRH sobre resultados de recursos humanos e o impacto indireto sobre os demais resultados. Segundo eles, GRH é o principal meio pelo qual o gerenciamento de uma organização pode comunicar suas metas, prioridades, e exigências. Por meio de suas práticas, GRH envia sinais para todos os recursos humanos da organização sobre os comportamentos e/ou resultados esperados e avaliados. Esses resultados interagem com os outros recursos e inputs funcionais da organização (dos outros processos) para criar resultados organizacionais.

Desse modo, os resultados organizacionais não são determinados apenas por resultados de recursos humanos. Ao invés disso, os resultados organizacionais são o produto da interação dos atuais resultados de recursos humanos com os outros recursos e inputs funcionais, desdobrados e usados pela organização. Ademais, os outros recursos e inputs funcionais da organização podem ser influenciados por stakeholders externos. Por sua vez, resultados financeiros e de mercado de capital são um produto da interação dos resultados organizacionais produzidos com as interpretações dos stakeholders externos.

Pelo exposto, e principalmente para aqueles pesquisadores que levam em consideração a perspectiva de múltiplos stakeholders em seu construto de desempenho organizacional, os quatro outcomes devem ser avaliados igualmente e em conjunto. Em tentativa de conciliação entre o modelo de Dyer e Reeves (1995), as descobertas de Combs et al. (2006) e a definição de desempenho 
organizacional como construto multidimensional (Rogers \& Wright, 1998; Way \& Johnson, 2005), concebemos a segunda hipótese, explicitada em seguida.

H2: O tamanho do efeito estimado para a relação entre práticas e sistemas de GRH será maior, quando dois ou mais destes resultados foram avaliados em conjunto do que quando foram avaliados em separado.

\section{Método}

\section{Procedimento de busca}

O processo de busca, da busca preliminar à busca final, teve duração de mais de um ano; foi iniciado em outubro de 2006 e concluído em novembro de 2007. Preliminarmente, utilizamos a busca de lista de referências em papers de revisão e de não revisão, em livros, ou em bibliografia especializada. Sete listas de referências foram selecionadas e consultadas por explicitarem sua amostra e fornecerem uma lista de suas unidades de análise. São cinco listas de referências provenientes de revisões narrativas (Boselie, Dietz, \& Boon, 2005; Huselid, 2003; Wall \& Wood, 2005; Wood, 1999a; Wright et al., 2005). E duas listas de referências provenientes de metanálises (Combs et al., 2006; Oliveira, 2006). Deste procedimento preliminar localizamos 265 referências de estudos primários relevantes e diferentes, realizados e/ou publicados até o ano de 2005, e publicados em língua inglesa.

Com o objetivo de evitar e/ou eliminar possíveis vieses advindos dos autores de cada uma destas listas de referências, como também de ampliar a amostra e identificar estudos realizados e/ou publicados entre os anos de 2005 e 2007, realizamos uma busca em índices de assunto de abstracts em bases de dados eletrônicas. As bases de dados que selecionamos, por suas relevâncias no tema, para identificar os estudos primários de interesse foram: (a) ISI Web of Knowledge que inclui a Web of Science, a Current Contents Connect e a ISI Proceedings; (b) Econlit e PsycInfo, compreendidas na ERL WebSPIRS 5; (c) EBSCOHOST que contém a Academic Search Premier; a Business Source Premier; a Regional Business News; e a Library, Information Science \& Technology; e, (d) ProQuest Dissertations and Theses composta pela Dissertations \& Theses e pela ABI/INFORM Global.

Os termos de busca human resource management e firm performance aparecem tanto nos títulos quanto nos resumos de estudos empíricos chaves sobre o tema (Delaney \& Huselid, 1996; Delery \& Doty, 1996; Huselid, 1995; Huselid, Jackson, \& Schuler, 1997; Youndt, Snell, Dean, \& Lepak, 1996). E, os termos: human resource management e organizational effectiveness são thesaurus nas bases de dados PsicInfo e ProQuest Dissertations and Theses; e são usados para substituir os termos: personnel management or human resources e organizational performance respectivamente. Desta forma, estabelecemos os seguintes termos de busca comuns para as quatro bases de dados: human resource management or high performance work practices and organizational effectiveness or firm performance, com limite temporal compreendido entre 1980 e 2007.

O resultado do somatório das referências identificadas nas listas de referências e das referências capturadas nestas bases de dados, depois de retiradas as referências duplicadas, foi de 1.392 referências diferentes. Contudo, ainda que algumas destas bases contenham material de outras línguas além da inglesa, todas as referências capturadas até este momento foram de língua inglesa.

A fim de incluir também material de língua portuguesa e espanhola, e diminuir o viés do material escrito em língua inglesa, identificamos, selecionamos e adicionamos mais quatro bases de dados nesta estratégia de busca: Banco de Teses do Portal de Periódicos da CAPES/MEC e Scientific Electronic Library Online (SCIELO Brasil) e, Ciências Sociales (ISOC) y Humanidades e Tesis Españolas (TESEO). Em cada uma destas bases utilizamos os termos de busca sugeridos pelas próprias bases. Assim, adicionamos 175 novas referências às referências já localizadas. 
Para complementarmos e finalizarmos a estratégia de busca, enviamos e-mails para os primeiros autores de estudos identificados nos outros procedimentos de busca, solicitando indicação de referências e/ou envio de texto completo de registros de estudos sobre o tema. No entanto somente dois autores forneceram referências de material não identificado pelos outros procedimentos de busca. Deste modo, identificamos ao todo 1.569 referências de registros de estudos diferentes.

Depois da identificação dessas referências, avaliamos a inclusão, ou não, de cada estudo nesta metanálise, de acordo com os critérios de elegibilidade. Os critérios que nós utilizamos para definir a elegibilidade dos estudos foram: (a) ser o registro de pelo menos um estudo primário de pesquisa empírica; (b) ter em suas medidas uma ou mais de práticas de GRH; (c) ter em suas medidas um ou mais indicadores de desempenho organizacional; (d) oferecer em seus resultados informes estatísticos suficientes (análise quantitativa) para o cálculo do tamanho do efeito entre as duas variáveis; (e) estar escrito em inglês, português ou espanhol; (f) oferecer dados originais que não se repetem em outro estudo; e (g) ter sido realizado e/ou publicado durante a década de 1980 ou depois

A análise dos critérios de inclusão e de exclusão de cada registro de estudo foi realizada em três etapas: (a) análise do abstract de todas as referências identificadas na estratégia de busca; (b) busca do texto completo das referências pré-selecionadas; e, (c) análise do texto completo de todas as referências pré-selecionadas e localizadas. No final, localizamos o texto completo e selecionamos 191 registros de estudos para compor a amostra deste estudo, por atenderem a todos os critérios de elegibilidade. Devido ao fato de que três registros de estudos, na realidade, apresentavam cada um dados de dois estudos diferentes (Allen, Shore, \& Griffeth, 2003; Tzafrir \& Eitam-Meilik, 2005; Zacharatos et al., 2005) dividimos estes três registros em dois cada um. Por este motivo, a amostra final desta metanálise foi de 194 registros de estudos.

\section{Procedimento de codificação}

De posse dos textos completos das 191 referências, iniciamos a preparação para o processo de codificação das variáveis. Esse processo foi realizado por dois codificadores. A fim de controlar e/ou reduzir os erros que são inerentes ao processo de codificação, realizamos alguns passos preliminares antes da codificação propriamente dita, como a elaboração de um Livro de Códigos e a criação de uma sequência aleatória das 191 referências para cada codificador. Depois, operacionalizamos o treinamento dos codificadores com um teste piloto, onde estimamos o tempo empreendido para codificar em média cada estudo, retiramos as dúvidas e as informações desnecessárias, contidas no Livro de Códigos. Por fim, realizamos o processo de codificação das variáveis, propriamente dito.

A medida do tamanho do efeito que nós elegemos para esta investigação foi o coeficiente de correlação de Pearson (r). Ademais, definimos a variável GRH como conjunto integrado de estratégias, políticas e práticas planejadas e intencionadas para gerenciar pessoas em uma organização (Martín-Alcázar, Romero-Fernández, \& Sánchez-Gardey, 2005; Wright \& McMahan, 1992). E, concebemos práticas de GRH como as atividades realmente implantadas e experimentadas pelos empregados, e que podem ser objetivamente verificadas (Arthur \& Boyles, 2007; Wright \& Nishii, 2006).

Para a operacionalização desta medida, inicialmente consideramos 17 práticas de GRH. A saber: (a) análise de cargo; (b) desenho de cargo; (c) planejamento de recursos humanos; (d) staffing externa; (e) staffing interna; (f) gestão do desligamento; (g) treinamento e desenvolvimento; (h) avaliação de desempenho; (i) compensação; (j) saúde, higiene e segurança no trabalho; (k) relações sindicais; (l) relações com o empregado; (m) gestão da diversidade; (n) sistemas de equipe de trabalho; (o) auditoria de GRH; (p) sistema de informação de GRH; (q) mudança e desenvolvimento organizacional.

Por sua vez, definimos Desempenho organizacional como um construto multidimensional que demonstra a efetividade de uma organização de acordo com os resultados apresentados: (a) os resultados de recursos humanos; (b) os resultados organizacionais; os (c) resultados financeiros ou contábeis; e/ou (d) os resultados de mercado de capital (Dyer \& Reeves, 1995; Ferris et al., 1998; 
Rogers \& Wright, 1998; Way \& Johnson, 2005). Assim, para efeito deste trabalho consideramos os quatro tipos de resultados e seus respectivos indicadores de desempenho organizacional.

Os resultados de recursos humanos, que incluem os indicadores de motivação, compromisso organizacional, satisfação no trabalho, desempenho individual, KSA (Knowledge, Skill, Ability), turnover (voluntário e/ou involuntário) e absenteísmo. Os resultados organizacionais, que envolvem índices de produtividade laboral, melhoria da eficiência (redução de custos laborais), melhoria da qualidade, satisfação do cliente, inovação e desenvolvimento de novos produtos e clima organizacional. Os resultados financeiros, tais como retorno sobre investimento, retorno sobre ativos e lucratividade ou rentabilidade contábil. E, por fim, os resultados de mercado, como preço de ações no mercado e Q de Tobin.

Logo após o término da coleta de dados, comparamos os dados e avaliamos a confiabilidade entre os codificadores. A confiabilidade estimada de cada dado foi tratada independentemente. Ademais, para avaliar a confiabilidade do processo de codificação dos dados, utilizamos três índices específicos de confiabilidade intercodificadores (IRR): o índice de acordo (AR), o Kappa de Cohen (k) e a correlação intercodificadores (r). Para avaliarmos cada índice estimado, seguimos as diretrizes de Orwin (1994) para os índices k e r, e arbitramos um valor mínimo para AR.

Desse modo, consideramos os três índices como adequados, o valor de AR foi superior a ,90; o valor estimado de $\mathrm{k}$ foi acima de, 75 ; e, o valor de $\mathrm{r}$ foi superior a ,80. Assim cumprimos com os nossos objetivos iniciais de controlar e/ou reduzir erros de informes de estudos primários deficientes, ambiguidade de julgamento e viés do codificador, bem como os erros de codificação propriamente ditos. Depois dessa análise, para cada dado em desacordo, independentemente de qual tenha sido o índice de confiabilidade intercodificadores estimado, retornamos aos textos completos dos estudos, a fim de estabelecer consenso.

Quando terminamos o processo de codificação, agrupamos as variáveis codificadas de acordo com a categorização das variáveis envolvidas e a frequência em que foram identificadas nos estudos. Deste modo, além das 17 práticas de GRH propostas inicialmente, a prática de segurança de emprego foi analisada, com uma frequência de $12 \%$, como uma variável de práticas de GRH pelos autores em seus estudos. Por este motivo, adicionamos esta prática à lista de práticas de GRH.

As práticas de GRH propostas inicialmente que tiveram também uma frequência maior ou igual que $10 \%$ foram: compensação $(43 \%)$ e, dentro destas, a prática de pagamento por desempenho, com $13 \%$; treinamento e desenvolvimento (37\%); mudança e desenvolvimento organizacional $(27 \%)$; desenho de cargo (19\%); staffing externa (19\%); avaliação de desempenho (16\%); staffing interna (16\%); sistema de trabalhos em equipe (13\%); e, relações com o empregado (11\%). As práticas de planejamento de recursos humanos, análise de cargo, gestão do desligamento, relações sindicais, sistema de informação de GRH, gestão da diversidade, auditoria de GRH e saúde, higiene e segurança no trabalho tiveram uma frequência igual ou menor que $5 \%$.

Importante observar que 55\% dos estudos agruparam práticas de GRH em um sistema ou configurações de práticas. Ademais, 34 estudos mediram e apresentaram resultados tanto de práticas de GRH de maneira isolada quanto de sistemas ou configurações de práticas de GRH. Isto equivale a $18 \%$ de todos os estudos analisados.

Quanto à variável desempenho organizacional, apenas 1\% dos 194 estudos estimou a correlação das práticas de GRH com indicadores de desempenho organizacional de todos os resultados. Enquanto $43 \%$ de todos os artigos investigaram exclusivamente a correlação das práticas de GRH com indicadores de resultados de recursos humanos, 53\% mediram esta correlação somente para os indicadores de resultados organizacionais; $40 \%$ investigaram a correlação das práticas de GRH apenas com os indicadores de resultados financeiros e, somente $8 \%$ analisaram esta correlação em relação aos indicadores de resultados de mercado.

Exploramos as variáveis codificadas e identificadas em cada estudo, a fim de encontrar semelhanças entre as correlações e seus respectivos tamanhos de efeito que possibilitassem a execução 
de uma metanálise. Como resultado desta exploração, identificamos dois agrupamentos para compor cada metanálise.

O primeiro agrupamento foi um tamanho de efeito para cada tipo de correlação em cada estudo. Dessa forma, em cada estudo, identificamos os diferentes tipos de correlações entre práticas de GRH e resultados de desempenho organizacional e calculamos, por média aritmética, um r para cada uma. No total, estimamos 887 coeficientes de correlação de Pearson. No entanto, como 34 estudos apresentaram resultados tanto de práticas isoladas de GRH quanto de sistemas de GRH, excluímos as correlações provenientes destes estudos, equivalentes a 297 correlações, para evitar dependência de dados. Assim, este primeiro agrupamento, ao qual denominamos de Agrupamento A, foi finalizado com 590 correlações.

O segundo agrupamento foi um coeficiente de correlação de Pearson para cada estudo. Para este agrupamento estimamos a média aritmética dos tamanhos do efeito obtidos com uma mesma amostra e incluímos esta estimação para análise. Novamente, para garantir a interdependência de dados, excluímos os 34 estudos que apresentaram resultados tanto de práticas isoladas de GRH quanto de sistemas de GRH. Desta maneira, este agrupamento, ao qual denominamos de Agrupamento B, finalizou com 160 estudos.

\section{Procedimentos metanalíticos}

Para a realização desta metanálise escolhemos o método de metanálise psicométrica de Hunter e Schmidt $(1990,2004)$ e corrigimos os artefatos com erro amostral e os artefatos com erro de medição das variáveis práticas de GRH e de desempenho organizacional. Todos os estudos analisados disponibilizaram informações para a correção do artefato com erro amostral. No entanto somente alguns estudos disponibilizaram informações para a correção dos artefatos com erro de medição da variável x (práticas de GRH) e/ou da variável y (indicadores de desempenho organizacional). Por este motivo, realizamos a metanálise de correlações por distribuições de artefatos recomendada por Hunter e Schmidt (2004).

Para a realização de uma metanálise por distribuição de artefatos, foram necessários dois estágios. Cada estágio correspondeu às informações disponibilizadas para cada artefato. No primeiro estágio, corrigimos individualmente o valor de $\mathrm{r}$ para o artefato com erro amostral. No segundo estágio corrigimos por distribuições os artefatos com erro de medição das variáveis x e y.

Depois do processamento dos dados, iniciamos a interpretação dos resultados. Primeiro verificamos a existência de indicadores de heterogeneidade. Quando positivo, analisamos a existência de variáveis moderadoras que incidem sobre a relação GRH-desempenho organizacional. Seguimos as recomendações de Cortina (2003) e utilizamos como critérios, além da regra de $75 \%$ de Hunter e Schmidt (2004), o desvio padrão das correlações (SDp) e o teste do $\mathrm{X}^{2}$. Já para o teste das variáveis moderadoras calculamos a média do desvio padrão dos agrupamentos (AveSDp), comparamos o limite mínimo, $\left(\mathrm{CI} 95 \%_{\min }\right)$ e o limite máximo, $\left(\mathrm{CI} 95 \%_{\max }\right)$ dos means de cada agrupamento e verificamos, por meio de representação gráfica, se entre os means de cada subagrupamento havia sobreposição de valores, tanto no limite mínimo quanto no limite máximo. Por fim, para complementarmos a análise das variáveis moderadoras, calculamos o mean padronizado da diferença do tamanho do efeito ( $\mathrm{g}$ ).

\section{Resultados}

Estimamos a magnitude ou tamanho do efeito da relação entre práticas de GRH e desempenho organizacional, por meio de uma metanálise com os 590 coeficientes de correlação de Pearson do Agrupamento A. Os resultados desta metanálise, sintetizados na Tabela 1, indicam que, com índice de confiança de 95\%, o valor estimado do coeficiente de correlação entre práticas de GRH e desempenho organizacional, depois da correção de artefatos com erro amostral e com erro de medição das variáveis 
GRH e desempenho organizacional, está compreendido entre ,203 e ,169; contudo, o valor que melhor o representa é: ,186; com nível de significância menor que ,010 ou igual.

Tabela 1

\section{Síntese dos Resultados do Agrupamento A}

\begin{tabular}{|c|c|c|c|c|c|c|c|c|c|c|c|}
\hline & \multirow[t]{2}{*}{$\mathbf{K}$} & \multirow[t]{2}{*}{$\sigma^{2} \mathbf{r}$} & \multirow[t]{2}{*}{$\sigma^{2} e$} & \multirow[t]{2}{*}{$\begin{array}{l}\text { Var } \\
\text { (p) }\end{array}$} & \multirow[t]{2}{*}{ SDp } & \multirow[t]{2}{*}{$\mathbf{p}$} & \multirow[t]{2}{*}{$\alpha$} & \multirow[t]{2}{*}{$\begin{array}{c}\text { \% de } \\
\text { S1 }^{2}\end{array}$} & \multirow[t]{2}{*}{$\begin{array}{c}\text { Q- } \\
\text { value }\end{array}$} & \multicolumn{2}{|c|}{$\begin{array}{c}95 \% \text { Intervalo } \\
\text { de } \\
\text { Confiança }\end{array}$} \\
\hline & & & & & & & & & & Máximo & Mínimo \\
\hline Agrupamento A & 590 & 0,044 & 0,002 & 0,068 & 0,262 & 0,186 & $<0,010$ & 0,960 & 12980 & 0,203 & 0,169 \\
\hline Práticas de GRH isoladas & 473 & 0,049 & 0,002 & $\mathbf{0 , 0 7 7}$ & 0,277 & 0,181 & $<0,010$ & 0,960 & 11589 & 0,201 & 0,161 \\
\hline Sistema de trabalho em equipe & 28 & 0,074 & 0,002 & 0,116 & 0,341 & 0,377 & $<0,050$ & 0,960 & 1036 & 0,478 & 0,276 \\
\hline Segurança de emprego & 23 & 0,065 & 0,002 & 0,102 & 0,319 & 0,338 & $<0,200$ & 0,960 & 748 & 0,442 & 0,234 \\
\hline Mudança e desenv. Org. & 50 & 0,047 & 0,002 & 0,073 & 0,271 & 0,294 & $<0,050$ & 0,960 & 1175 & 0,354 & 0,234 \\
\hline Pagamento por desempenho & 32 & 0,053 & 0,001 & 0,083 & 0,289 & 0,262 & $<0,200$ & 0,980 & 1696 & 0,342 & 0,182 \\
\hline $\begin{array}{l}\text { Treinamento e } \\
\text { desenvolvimento }\end{array}$ & 76 & 0,038 & 0,002 & 0,059 & 0,242 & 0,210 & $<0,100$ & 0,950 & 1444 & 0,254 & 0,166 \\
\hline Desenho de cargo & 34 & 0,050 & 0,001 & 0,078 & 0,280 & 0,191 & ns & 0,970 & 1700 & 0,266 & 0,116 \\
\hline Avaliação de desempenho & 38 & 0,060 & 0,004 & 0,092 & 0,304 & 0,183 & ns & 0,940 & 570 & 0,261 & 0,105 \\
\hline Staffing externa & 36 & 0,033 & 0,004 & 0,047 & 0,217 & 0,169 & ns & 0,870 & 297 & 0,228 & 0,110 \\
\hline Compensação & 66 & 0,011 & 0,001 & 0,016 & 0,126 & 0,135 & ns & 0,900 & 726 & 0,160 & 0,110 \\
\hline Relações com o empregado & 18 & 0,056 & 0,003 & 0,087 & 0,295 & 0,107 & ns & 0,950 & 336 & 0,216 & $-0,002$ \\
\hline Staffing interna & 40 & 0,086 & 0,002 & 0,138 & 0,371 & 0,097 & ns & 0,980 & 1720 & 0,188 & 0,006 \\
\hline Sistemas de GRH & 117 & 0,018 & 0,002 & 0,025 & 0,158 & 0,210 & $<0,010$ & $\mathbf{0 , 8 7 0}$ & 1053 & 0,234 & 0,186 \\
\hline Resultados conjuntos & 92 & 0,047 & 0,004 & $\mathbf{0 , 0 7 0}$ & 0,264 & 0,224 & $<\mathbf{0 , 0 5 0}$ & 0,910 & 1081 & 0,268 & 0,180 \\
\hline Resultados individuais & 498 & 0,044 & 0,002 & 0,068 & 0,261 & 0,184 & $<0,010$ & 0,960 & 10956 & 0,202 & 0,166 \\
\hline
\end{tabular}

Nota. $\mathrm{K}=$ número de correlações; $\sigma^{2} \mathrm{r}=$ variância observada entre estudos; $\sigma^{2} \mathrm{e}=$ variância entre estudos devido ao erro amostral; $\operatorname{Var}(\mathrm{p})$ = variância estimada depois da correção de todos os artefatos com informação disponível; SDp = desvio padrão real das correlações; $\mathrm{p}=$ mean da correlação da população real; $\alpha=$ nível de significância; $\%$ de $\mathrm{S} 1{ }^{2}=$ percentual da variância em correlações produzidas pela variação das correlações reais e não corrigidas em nenhum estágio da metanálise em relação a $\sigma^{2}$ r; Q-value $=$ teste do $\mathrm{X}^{2}$.

Depois de estimarmos a magnitude da relação entre as variáveis, avaliamos e identificamos quais práticas de GRH se correlacionam mais com desempenho organizacional de forma geral. Para tanto, realizamos uma metanálise para cada agrupamento de práticas de GRH que obtiveram uma frequência maior que $10 \%$ ou igual. Assim, na ordem apresentada, as práticas: sistema de trabalhos em equipe $(\mathrm{k}=28 ; \mathrm{p}=, 377 ; \alpha \leq, 050)$; segurança de emprego $(\mathrm{k}=23 ; \mathrm{p}=, 338 ; \alpha \leq, 200)$; mudança $\mathrm{e}$ desenvolvimento organizacional $(\mathrm{k}=50 ; \mathrm{p}=, 294 ; \alpha \leq, 050)$; pagamento por desempenho $(\mathrm{k}=32 ; \mathrm{p}=$ ,262; $\alpha \leq, 200)$; treinamento e desenvolvimento $(\mathrm{k}=76 ; \mathrm{p}=, 210 ; \alpha \leq, 100)$; e, desenho de cargo $(\mathrm{k}=$ $34 ; \mathrm{p}=, 191 ; \mathrm{ns})$ apresentaram um mean superior ao mean de todas as correlações da população $(\mathrm{k}=$ $590 ; \mathrm{p}=, 186 ; \alpha \leq, 010)$.

Por outro lado, as práticas: avaliação de desempenho $(\mathrm{k}=38 ; \mathrm{p}=, 183$; ns); staffing externa $(\mathrm{k}=$ $36 ; \mathrm{p}=, 169 ; \mathrm{ns})$; compensação $(\mathrm{k}=66 ; \mathrm{p}=, 135 ; \mathrm{ns})$; relações com o empregado $(\mathrm{k}=18 ; \mathrm{p}=, 107$; $\mathrm{ns})$; e, staffing interna $(\mathrm{k}=40 ; \mathrm{p}=, 097 ; \mathrm{ns})$ apresentaram um mean não tão satisfatório. Ademais, o intervalo de confiança da prática relações com o empregado mostra um valor mínimo abaixo de zero; isto significa que a relação desta prática com o desempenho organizacional pode ser negativa. 
Para avaliarmos se um sistema de GRH se correlaciona mais com desempenho organizacional em geral do que com cada prática separada, dividimos as 590 correlações em dois grupos, o grupo das correlações observadas entre sistemas de GRH e desempenho organizacional e o grupo das correlações observadas entre todas as práticas isoladas de GRH e desempenho organizacional. Os resultados desta divisão demonstram que o mean de sistemas de GRH $(\mathrm{k}=117 ; \mathrm{p}=, 210 ; \alpha \leq, 010)$ é maior do que o mean de práticas de GRH isoladas $(\mathrm{k}=473 ; \mathrm{p}=, 181 ; \alpha \leq, 010)$. O Ave(SDp) dos dois grupos é menor do que o SDp conjunto; ,218 e 262 respectivamente. Ademais, o intervalo de confiança entre os dois grupos apresenta uma sobreposição entre o limite mínimo de sistemas de GRH e o limite máximo de práticas de GRH isoladas. Estes resultados indicam a possibilidade de variável moderadora. No entanto a diferença entre os means dos dois grupos é considerada pequena $(g=, 111)$.

Na sequência, avaliamos se um sistema de GRH se correlaciona mais com cada resultado específico do que com cada prática em separado; cada um dos dois grupos, sistemas de GRH e práticas de GRH isoladas, foi desmembrado para cada resultado de desempenho organizacional. Em relação aos resultados de recursos humanos, o mean de sistemas de GRH $(\mathrm{k}=30 ; \mathrm{p}=, 210$; ns) é sutilmente maior do que o mean de práticas de GRH isoladas $(\mathrm{k}=141 ; \mathrm{p}=, 193 ; \alpha \leq, 010)$. Também, o Ave(SDp) dos dois grupos; ,187 é menor do que o SDp; ,193. Contudo o intervalo de confiança entre os dois grupos mostra uma sobreposição total dos valores estimados, que foi reforçada por um valor padronizado da diferença muito pequeno $(\mathrm{g}=, 088)$.

Para os resultados organizacionais, os dados não foram diferentes, o mean de sistemas de GRH $(\mathrm{k}=38 ; \mathrm{p}=, 207 ; \alpha \leq, 200)$ é quase o mesmo do mean de práticas de GRH isoladas $(\mathrm{k}=135 ; \mathrm{p}=, 203$; $\alpha \leq, 050$ ). Embora, o Ave(SDp) dos dois grupos; ,254 seja menor do que o SDp; ,309, estes dados se confirmam com a sobreposição de means apresentada no intervalo de confiança entre os dois grupos e o valor insignificante da diferença entre esses means $(g=, 013)$.

Para os resultados financeiros, o mean de sistemas de GRH ( $k=30 ; \mathrm{p}=, 193 ; \mathrm{ns})$, como para os resultados de recursos humanos, é sutilmente maior do que o mean de práticas de GRH isoladas $(\mathrm{k}=$ $108 ; \mathrm{p}=, 157 ; \alpha \leq, 200)$. Novamente, o Ave(SDp) dos dois grupos; 207 é menor do que o SDp; ,285; no entanto a sobreposição apresentada no intervalo de confiança entre os means dos dois grupos e o pequeno valor da diferença entre seus means $(g=, 126)$ reforçaram a impressão inicial.

Em contrapartida, para os resultados de mercado, o mean da diferença padronizada $(g=, 976)$ é considerado grande. Ademais, não há sobreposição de dados no intervalo de confiança entre os dois grupos. Ainda, para reforçar mais a diferença entre os means, o Ave(SDp) dos dois grupos; ,046, é muito menor do que o SDp; ,082. Os dados também demonstram que a correlação entre sistemas de GRH $(\mathrm{k}=7 ; \mathrm{p}=, 167 ; \mathrm{ns})$ e resultados de mercado é maior do que a correlação entre práticas de GRH isoladas e resultados de mercado $(\mathrm{k}=9 ; \mathrm{p}=, 087 ; \mathrm{ns})$. Contudo a baixa frequência de correlações deste tipo de resultado pode induzir uma falsa afirmação sobre a existência de um efeito moderador. Por este motivo, analisamos os dados para os resultados de mercado com cautela.

Por último, realizamos uma análise adicional com os dados do Agrupamento B, cujos resultados apresentamos na Tabela 2. Para tanto, dividimos este agrupamento em dois grupos, o grupo das correlações observadas entre sistemas de GRH e desempenho organizacional e o grupo das correlações observadas entre todas as práticas de GRH isoladas e desempenho organizacional. Os resultados desta divisão confirmam que o mean de sistemas de GRH $(\mathrm{k}=74 ; \mathrm{p}=, 224 ; \alpha \leq, 050)$ é maior do que o mean de práticas de GRH isoladas $(\mathrm{k}=86 ; \mathrm{p}=, 143 ; \alpha \leq, 200)$. O Ave(SDp) dos dois grupos; ,166, é menor do que o SDp conjunto; ,168. O intervalo de confiança entre os dois grupos não apresenta sobreposição. Ademais, a diferença entre os means dos dois grupos é considerada mediana $(\mathrm{g}=, 482)$. 
Tabela 2

Síntese dos Resultados do Agrupamento B

\begin{tabular}{|c|c|c|c|c|c|c|c|c|c|c|c|}
\hline & \multirow[t]{2}{*}{$\mathbf{K}$} & \multirow[t]{2}{*}{$\sigma^{2} \mathbf{r}$} & \multirow[t]{2}{*}{$\sigma^{2} e$} & \multirow[t]{2}{*}{$\operatorname{Var}(\mathbf{p})$} & \multirow[t]{2}{*}{ SDp } & \multirow[t]{2}{*}{$\mathbf{p}$} & \multirow[t]{2}{*}{$\alpha$} & \multirow[t]{2}{*}{$\%$ de $S 1^{2}$} & \multirow[t]{2}{*}{ Q-value } & \multicolumn{2}{|c|}{$\begin{array}{l}\text { 95\% Intervalo de } \\
\text { Confiança }\end{array}$} \\
\hline & & & & & & & & & & Máximo & Mínimo \\
\hline Agrupamento B & 160 & 0,020 & 0,001 & 0,028 & 0,168 & 0,163 & $<0,050$ & 0,930 & 3200 & 0,185 & 0,141 \\
\hline Práticas de GRH isoladas & 86 & 0,018 & 0,001 & 0,026 & 0,162 & 0,143 & $<0,200$ & 0,940 & 1548 & 0,171 & 0,115 \\
\hline Sistemas de GRH & 74 & 0,021 & 0,002 & 0,029 & 0,170 & 0,224 & $<0,050$ & 0,880 & 777 & 0,257 & 0,191 \\
\hline Resultados individuais & 80 & 0,012 & 0,001 & 0,017 & 0,129 & 0,133 & ns & 0,900 & 960 & 0,157 & 0,109 \\
\hline Resultados conjuntos & 80 & 0,029 & 0,002 & 0,042 & 0,204 & 0,206 & $<0,100$ & 0,940 & 1160 & 0,243 & 0,169 \\
\hline
\end{tabular}

Nota. $\mathrm{K}=$ número de correlações; $\sigma^{2} \mathrm{r}=$ variância observada entre estudos; $\sigma^{2} \mathrm{e}=$ variância entre estudos devido ao erro amostral; $\operatorname{Var}(\mathrm{p})$ = variância estimada depois da correção de todos os artefatos com informação disponível; SDp $=$ desvio padrão real das correlações; $p=$ mean da correlação da população real; $\alpha=$ nível de significância; \% de S1 ${ }^{2}=$ percentual da variância em correlações produzidas pela variação das correlações reais e não corrigidas em nenhum estágio da metanálise em relação a $\sigma^{2}$ r; Q-value $=$ teste do $\mathrm{X}^{2}$.

Com base nos resultados apresentados até o momento, verificamos que o tamanho do efeito estimado para sistemas de GRH e desempenho organizacional foi maior do que o tamanho do efeito estimado para cada prática de GRH em separado e o desempenho organizacional. Assim, confirmamos a primeira hipótese.

Na sequência, para identificarmos e desenvolvermos um modelo com a variável moderadora de tipo substantivo e avaliarmos se o tamanho do efeito da relação entre práticas de GRH e desempenho organizacional varia em relação ao tipo de resultado de desempenho organizacional, dividimos as 590 correlações em outros dois grupos. Ou seja, um grupo foi constituído das correlações observadas entre práticas e sistemas de GRH e cada resultado medido separadamente, enquanto o outro grupo foi constituído pelas correlações observadas entre práticas e sistemas de GRH e resultados medidos conjuntamente. Assim, as correlações observadas para resultados de recursos humanos, resultados organizacionais, resultados financeiros ou resultados de mercado, constituíram o grupo dos resultados individuais. $\mathrm{E}$ as correlações observadas para dois, três ou os quatro resultados de desempenho organizacional constituíram o grupo dos resultados conjuntos.

Os resultados da metanálise realizada para cada grupo revelam que o mean de resultados conjuntos $(\mathrm{k}=92 ; \mathrm{p}=, 224 ; \alpha \leq, 050)$ é maior do que o mean de resultados individuais $(\mathrm{k}=498 ; \mathrm{p}=$ ,184; $\alpha \leq, 010)$. Ademais, o mean de resultados conjuntos também é maior do que o mean de cada resultado avaliado separadamente: recursos humanos $(\mathrm{k}=171 ; \mathrm{p}=, 196 ; \alpha \leq, 020)$, organizacional $(\mathrm{k}=$ $173 ; \mathrm{p}=, 204 ; \alpha \leq, 010)$, financeiro $(\mathrm{k}=138 ; \mathrm{p}=, 162 ; \alpha \leq, 050)$ e de mercado $(\mathrm{k}=16 ; \mathrm{p}=, 110 ; \mathrm{ns})$. No entanto o Ave(SDp) dos grupos resultados conjuntos e resultados individuais; ,263 é ligeiramente maior do que o SDp; ,262. O intervalo de confiança entre os dois grupos apresenta certa sobreposição de dados, reforçada pelo pequeno valor da diferença entre seus means $(g=, 153)$.

Devido a estes resultados contraditórios, realizamos uma análise adicional com o Agrupamento B. Para tanto, dividimos o Agrupamento B também em dois grupos: o grupo resultados individuais e o grupo resultados conjuntos. Os resultados da metanálise realizada para cada grupo revelam que o mean de resultados conjuntos $(\mathrm{k}=80 ; \mathrm{p}=, 206 ; \alpha \leq, 100)$ é maior do que o mean de resultados individuais $(\mathrm{k}=80 ; \mathrm{p}=, 133 ; \mathrm{ns})$. O Ave(SDp) dos grupos resultados conjuntos e resultados individuais; ,167 é menor do que o SDp; ,168. O intervalo de confiança entre os dois grupos não apresenta sobreposição de dados. Dessa forma o valor da diferença entre seus means é mediano ( $\mathrm{g}=$ ,435).

Os dados apresentados ratificam o que previamente estabelecemos no sentido de que o tamanho do efeito estimado para a relação entre práticas e sistemas de GRH será maior, quando dois ou mais 
destes resultados foram avaliados em conjunto do que quando foram avaliados em separado. Assim, confirmamos também a segunda hipótese.

\section{Discussões}

Os resultados das metanálises constituem-se nas ações realizadas para o atendimento dos objetivos propostos. Por sua vez, traduzimos estas ações nas pretensas contribuições deste trabalho: estimação da magnitude; identificação e desenvolvimento de modelos com variáveis moderadoras de tipo substantivo, para contribuir com o entendimento da relação entre práticas de GRH e desempenho organizacional.

Calculamos a estimação pontual de ,186 para a magnitude da relação entre práticas de GRH e desempenho organizacional. Esta estimação está entre as estimações das duas metanálises que foram realizadas recentemente (Combs et al., 2006; Oliveira, 2006). Para entendermos, e quiçá justificarmos, esta estimação, é importante lembrarmos que inúmeros fatores interferem na relação práticas de GRHdesempenho organizacional. Em complemento, de acordo com o argumento de Dyer e Reeves (1995), estes inúmeros fatores influenciam resultados ou outcomes que, por sua vez, diminuem a relativa contribuição de GRH para estes outcomes.

As práticas de GRH isoladas que mais se correlacionaram com desempenho organizacional em geral foram: as práticas de sistema de trabalhos em equipe; segurança de emprego; mudança e desenvolvimento organizacional; pagamento por desempenho; e, treinamento e desenvolvimento. Estes resultados mostram semelhanças e divergências com as duas metanálises realizadas anteriormente (Combs et al., 2006; Oliveira, 2006). Especificamente, as práticas de segurança de emprego e treinamento e desenvolvimento também reportaram os melhores resultados, em ambas. Por outro lado, as práticas de sistema de trabalhos em equipe e de mudança e desenvolvimento organizacional, que envolve a prática de informação compartilhada, também em ambas, apresentaram os piores índices.

Podemos justificar estas divergências pelas teorias de dependência de recursos e institucional. Estas teorias baseiam-se no argumento de que a variância apresentada em práticas de GRH não explicada pelo processo de tomada de decisão estratégica pode dever-se a forças políticas e institucionais (Wright \& McMahan, 1992). Isto significa que uma mesma prática pode ser experimentada e avaliada diferentemente, a depender das forças políticas e institucionais que permeiam uma organização.

No entanto os resultados também indicam que um sistema de GRH se correlaciona mais com desempenho organizacional em geral do que com práticas de GRH isoladas. Estes resultados apoiam a descoberta, a síntese e a descrição dos resultados de pesquisa já existentes (Combs et al., 2006; Oliveira, 2006). Além disso, reforçam os argumentos da teoria, baseada em recursos e capacidades, de que os efeitos das práticas de GRH, quando em combinação com outros recursos e capacidades, são maiores do que a soma de suas partes (Barney, 1995).

Depois, realizamos a identificação e desenvolvimento de modelos com variáveis moderadoras de tipo substantivo. Os resultados apresentados corroboram a suposição de que o tamanho do efeito estimado para a relação entre práticas e sistemas de GRH será maior, quando dois os mais resultados de desempenho organizacional foram avaliados em conjunto, do que quando foram avaliados em separado.

Estes resultados contradizem os resultados apresentados por Combs et al. (2006). Uma possível explicação para esta divergência é que Combs et al. só utilizaram um indicador de desempenho organizacional para cada resultado. Em contrapartida, confirmam o modelo proposto por Dyer e Reeves (1995) de que os outcomes de recursos humanos e os outcomes organizacionais podem servir de moderadores entre GRH e os outcomes financeiros e de mercado, porquanto, que em uma 
metanálise realizada com os quatro tipos de resultados de desempenho organizacional, as duas maiores correlações foram referentes aos grupos de resultados organizacionais e de resultados de recursos humanos.

Com base nestes resultados, podemos inferir que há relação positiva entre GRH e desempenho organizacional. A estimação da magnitude desta relação aumenta, quando as práticas de GRH são combinadas em um sistema, ou dois os mais resultados de desempenho organizacional são levados em consideração; portanto o ponto de vista configuracional parece ser o mais adequado para analisar o impacto da gestão de recursos humanos sobre o desempenho organizacional (Delery \& Doty, 1996).

Desde que as últimas revisões foram realizadas (Combs et al., 2006; Oliveira, 2006; Oliveira et al., 2007a, 2007b), o número de novos estudos empíricos que buscam elucidar a relação entre GRH e desempenho organizacional continuou a crescer. Este fato por si só justificou a realização de outra metanálise, a fim de atualizar seus resultados. Assim, 31 dos 191 registros de estudos selecionados para compor a amostra desta revisão metanalítica foram realizados e/ou publicados entre 2005 e 2007; em consequência, cumpre admitir que não foram analisados anteriormente.

Deste modo, a atualização da estimação da magnitude da relação e a identificação de variáveis moderadoras contribuem para o entendimento da relação entre GRH e desempenho organizacional; conseguintemente produzem implicações tanto teóricas quanto práticas. Em termos acadêmicos, estes resultados podem contribuir com investigações futuras, pois asseguram aos pesquisadores, por meio de evidências empíricas, que há relação significante e positiva entre as variáveis: práticas de GRH e desempenho organizacional. Em termos práticos, estes resultados, ademais de justificarem investimentos em GRH, oferecem aos profissionais diretrizes para uma atuação mais configuracional.

Com base nas reflexões apresentadas e contribuições deste trabalho, identificamos questões que carecem de mais investigação. A primeira questão refere-se à correção de artefatos. Outros artefatos que possivelmente estão presentes nos estudos que avaliam a relação entre medidas de práticas de GRH e medidas de desempenho organizacional, também precisam ser considerados e corrigidos. Tendo em vista que é provável que artefatos como restrição de intervalo, dicotomização de variáveis ou desvio para perfeita validade de constructo também podem interferir nos resultados destes estudos. A consideração e a correção de outros artefatos, além dos artefatos com erro amostral e com erro de medição podem proporcionar uma estimação da magnitude desta relação ainda mais precisa.

A segunda questão se refere aos descritores de estudos que o pesquisador deve considerar antecipadamente, para conduzir análises interessantes em relação aos seus resultados. Muitos descritores de estudos ainda não foram explorados. Estes descritores envolvem os fenômenos contextuais, os métodos de pesquisa e outros fatores extrínsecos aos dois primeiros. Como complemento, a terceira sugestão envolve a análise do relacionamento entre os descritores de estudos. A análise da intercorrelação entre descritores de estudos podem revelar clusters que não foram explorados, ademais de ser útil para sintetizar dados e, principalmente, identificar possíveis variáveis moderadoras.

Em suma, o campo de pesquisa que se refere à relação entre práticas de GRH e desempenho organizacional ainda apresenta inúmeras possibilidades de pesquisa. Algumas destas possibilidades já foram exploradas, mas carecem de atualização e outras não exploradas, no entanto, podem proporcionar descobertas inéditas.

Artigo recebido em 14.09.2010. Aprovado em 10.02.2011. 


\section{Referências}

Agarwala, T. (2003). Innovative human resource practices and organizational commitment: an empirical investigation. International Journal of Human Resource Management, 14(2), 175197. doi: 10.1080/0958519021000029072

Allen, D. G., Shore, L. M., \& Griffeth, R. W. (2003). The role of perceived organizational support and supportive human resource practices in the turnover process. Journal of Management, 29(1), 99118. doi: $10.1016 / \mathrm{S} 0149-2063(02) 00222-2$

Arthur, J. B., \& Boyles, T. (2007). Validating the human resource system structure: a levels-based strategic HRM approach. Human Resource Management Review, 17(1), 77-92. doi: 10.1016/j.hrmr.2007.02.001

Audea, T., Teo, S. T. T., \& Crawford, J. (2005). HRM professionals and their perceptions of HRM and firm performance in the Philippines. International Journal of Human Resource Management, 16(4), 532-552. doi: 10.1080/09585190500051589

Barney, J. B. (1995). Looking inside for competitive advantage. Academy of Management Executive, 9(4), 49-61. doi: 10.2307/4165288

Barrette, J., \& Ouellette, R. (2000). Gestion de la performance: impact sur la performance organisationnelle de l'intégration de la stratégie et de la cohérence des systèmes de GRH. Relations Industrielles, 55(2), 207-226.

Becker, B. E., \& Gerhart, B. (1996). The impact of human resource management on organizational performance: progress and prospects. Academy of Management Journal, 39(4), 779-801. doi: $10.2307 / 256712$

Becker, B. E., \& Huselid, M. A. (1998a). High performance work systems and firm performance: a synthesis of research and managerial implications. In G. R. Ferris (Ed.), Research in personnel and human resource management (Vol. 16, pp. 53-101). Oxford, England: JAI Press Inc.

Becker, B. E., \& Huselid, M. A. (1998b). Human resources strategies, complementarities, and firm performance. (Relatório de Pesquisa/1998), New Brunswick, NJ, School of Management and Labor Relations at Rutgers University and SUNY-Buffalo School of Management.

Boselie, P., Dietz, G., \& Boon, C. (2005). Commonalities and contradictions in HRM and performance research. Human Resource Management Journal, 15(3), 67-94. doi: 10.1111/j.1748-8583.2005.tb00154.x

Burton, M. D., \& O'Reilly, C. A., III (2000, August). The impact of high commitment values and practices on technology start-ups. Anais do Annual Meeting of the Academy of Management, Toronto, Canadá, 60.

Chang, E. (2005). Employees' overall perception of HRM effectiveness. Human Relations, 58(4), 523544. doi: $10.1177 / 0018726705055037$

Colakoglu, S., Lepak, D. P., \& Hong, Y. (2006). Measuring HRM effectiveness: considering multiple stakeholders in a global context. Human Resource Management Review, 16(2), 209-218. doi: 10.1016/j.hrmr.2006.03.003

Collins, C. J., \& Smith, K. G. (2006). Knowledge exchange and combination: the role of human resource practices in the performance of high-technology firms. Academy of Management Journal, 49(3), 544-560. doi: 10.2307/20159780

Combs, J., Liu, Y., Hall, A., \& Ketchen, D. (2006). How much do high-performance work practices 
matter? A meta-analysis of their effects on organizational performance. Personnel Psychology, 59(3), 501-528. doi: 10.1111/j.1744-6570.2006.00045.x

Cortina, J. M. (2003). Apples and oranges (and pears, oh my!): the search for moderators in metaanalysis. Organizational Research Methods, 6(4), 415-439. doi: 10.1177/1094428103257358

Delaney, J. T., \& Huselid, M. A. (1996). The impact of human resource management practices on perceptions of organizational performance. Academy of Management Journal, 39(4), 949-969. doi: $10.2307 / 256718$

Delery, J. E., \& Doty, D. H. (1996). Modes of theorizing in strategic human resource management: tests of universalistic, contingency, and configurational performance predictions. Academy of Management Journal, 39(4), 802-835. doi: 10.2307/256713

Den Hartog, D. N., \& Verburg, R. M. (2004). High performance work systems, organisational culture and firm effectiveness. Human Resource Management Journal, 14(1), 55-78. doi: $10.1111 / \mathrm{j} .1748-8583.2004 . t b 00112 . x$

Dyer, L. (1984). Studying human resource strategy: an approach and an agenda. Industrial Relations, 23(2), 156-169. doi: 10.1111/j.1468-232X.1984.tb00894.x

Dyer, L., \& Reeves, T. (1995). Human resource strategies and firm performance: what do we know and where do we need to go? International Journal of Human Resource Management, 6(3), 656-670. doi: 10.1080/09585199500000041

Ferris, G. R., Arthur, M. M., Berkson, H. M., Kaplan, D. M., Harrell-Cook, G., \& Frink, D. D. (1998). Toward a social context theory of the human resource management-organization effectiveness relationship. Human Resource Management Review, 8(3), 235-264. doi: 10.1016/S10534822(98)90004-3

Ferris, G. R., Hochwarter, W. A., Buckley, M. R., Harrell-Cook, G., \& Frink, D. D. (1999). Human resources management: some new directions. Journal of Management, 25(3), 385-415. doi: 10.1016/S0149-2063(99)00007-0

Gould-Williams, J. (2004). The effects of 'high commitment' HRM practices on employee attitude: the views of public sector workers. Public Administration, 82(1), 63-81. doi: 10.1111/j.00333298.2004.00383.x

Guerrero, S., \& Barraud-Didier, V. (2004). High-involvement practices and performance of French firms. International Journal of Human Resource Management, 15(8), 1408-1423. doi: $10.1080 / 0958519042000258002$

Guest, D. E. (1997). Human resource management and performance: a review and research agenda. International Journal of Human Resource Management, 8(3), 263-276. doi: $10.1080 / 095851997341630$

Guthrie, J. P. (2001). High-involvement work practices, turnover, and productivity: evidence from New Zealand. Academy of Management Journal, 44(1), 180-190. doi:10.2307/3069345

Guthrie, J. P., Datta, D. K., \& Wright, P. M. (2004). Peeling back the onion competitive advantage through people: test of a causal model [Working Paper $\mathrm{N}^{\circ}$ 15]. Cornell University, Ithaca, NY, USA.

Guthrie, J. P., Spell, C. S., \& Nyamori, R. O. (2002). Correlates and consequences of high involvement work practices: the role of competitive strategy. International Journal of Human Resource Management, 13(1), 183-197. doi: 10.1080/09585190110085071

Guzzo, R. A., Jette, R. D., \& Katzell, R. A. (1985). The effects of psychologically based intervention 
programs on worker productivity: a meta-analysis. Personnel Psychology, 38(2), 275-291. doi: 10.1111/j.1744-6570.1985.tb00547.x

Hunter, J. E., \& Schmidt, F. L. (1990). Methods of meta-analysis: correcting error and bias in research findings. Newburry Park, CA: Sage Publications.

Hunter, J. E., \& Schmidt, F. L. (2004). Methods of meta-analysis: correcting error and bias in research findings ( $2 \mathrm{a}$ ed.). Thousand Oaks, CA: Sage Publications.

Huselid, M. A. (1995). The impact of human resource management practices on turnover, productivity, and corporate financial performance. Academy of Management Journal, 38(3), 635-672. doi: 10.2307/256741

Huselid, M. A. (2003). HR management - firm performance empirical papers: 1995-2003. Recuperado em 15 janeiro, 2006, de http://www.markhuselid.com/pdfs/.../2003_SHRM_References_9503.pdf

Huselid, M. A., \& Becker, B. E. (1995). The strategic impact of high performance work systems (Relatório de Pesquisa/1995), New Brunswick, NJ, Human Resource Planning Society, Society for Human Resource Management Foundation, School of Management and Labor Relations at Rutgers University, and SUNY-Buffalo School of Management.

Huselid, M. A., Jackson, S. E., \& Schuler, R. S. (1997). Technical and strategic human resource management effectiveness as determinants of firm performance. Academy of Management Journal, 40(1), 171-188. doi: 10.2307/257025

Ichniowski, C., Kochan, T. A., Levine, D. I., Olson, C. A., \& Strauss, G. (1996). What works at work: overview and assessment. Industrial Relations, 35(3), 299-333. doi: 10.1111/j.1468232X.1996.tb00409.x

Ichniowski, C., \& Shaw, K. (1999). The effects of human resource management systems on economic performance: an international comparison of U.S. and Japanese plants. Management Science, 45(5), 704-721. doi: 10.1287/mnsc.45.5.704

Koys, D. J. (2001). The effects of employee satisfaction, organizational citizenship behavior, and turnover on organizational effectiveness: a unit-level, longitudinal study. Personnel Psychology, 54(1), 101-114. doi: 10.1111/j.1744-6570.2001.tb00087.x

Lacombe, B. M. B. (2004). Avaliação de resultados em $R H$ : análise dos métodos propostos na literatura acadêmica. (Relatório de Pesquisa n ${ }^{\circ} 29$ ), São Paulo, SP, Escola de Administração de Empresas de São Paulo, Fundação Getúlio Vargas.

Liouville, J., \& Bayad, M. (1998). Human resource management and performances: proposition and test of a causal model. Human Systems Management, 17(3), 183-192.

Macky, K., \& Boxall, P. (2007). The relationship between 'high-performance work practices' and employee attitudes: an investigation of additive and interaction effects. International Journal of Human Resource Management, 18(4), 537-567. doi: 10.1080/09585190601178745

Macky, K., \& Boxall, P. (2008). High-involvement work processes, work intensification and employee well-being: a study of New Zealand worker experiences. Asia Pacific Journal of Human Resources, 46(1), 38-55. doi: 10.1177/1038411107086542

Martín-Alcázar, F., Romero-Fernández, P. M., \& Sánchez-Gardey, G. (2005). Strategic human resource management: integrating the universalistic, contingent, configurational and contextual perspectives. International Journal of Human Resource Management, 16(5), 633-659. doi: $10.1080 / 09585190500082519$ 
Oliveira, A. M. B. de (2006). A relação entre as práticas de recursos humanos e o desempenho organizacional: uma metanálise (Trabalho de conclusão de curso não publicado). Universidad de Salamanca, Salamanca, España.

Oliveira, A. M. B. de, González, L. F., \& Oliveira, A. J. (2007a, May). The design investigation as a variable moderator in the study of the relationship between practices of HR and organizational performance. Anais do European Congress of Work and Organizational Psychology, Estocolmo, Suécia, 13.

Oliveira, A. M. B. de, González, L. F., \& Oliveira, A. J. (2007b, May). The human resource management and the organizational performance: one meta-analysis of the proposal of Pfeffer. Anais do European Congress of Work and Organizational Psychology, Estocolmo, Suécia, 13.

Orwin, R. G. (1994). Evaluating coding decisions. In H. Cooper \& L. V. Hedges (Eds.), The handbook of research synthesis (pp. 139-162). New York: Russell Sage Foundation.

Paauwe, J., \& Boselie, P. (2005). HRM and performance: what next? Human Resource Management Journal, 15(4), 68-83. doi: 10.1111/j.1748-8583.2005.tb00296.x

Paul, A. K., \& Anantharaman, R. N. (2003). Impact of people management practices on organizational performance: analysis of a causal model. International Journal of Human Resource Management, 14(7), 1246-1266. doi: 10.1080/0958519032000145648

Pfeffer, J. (1998). Seven practices of successful organizations. California Management Review, 40(2), 96-124.

Rogers, E. W., \& Wright, P. M. (1998). Measuring organizational performance in strategic human resource management: problems, prospects, and performance information markets. Human Resource Management Review, 8(3), 311-331. doi: 10.1016/S1053-4822(98)90007-9

Sanz-Valle, R., \& Sánchez, R. S. (2000). Efectos del enfoque estratégico de recursos humanos en los resultados de la empresa. Revista de Trabajo y Seguridad Social: Estudios Financieros (Recursos Humanos), 211(44), 167-204.

Schuler, R. S., \& Jackson, S. E. (1987). Linking competitive strategies with human resource management practices. Academy of Management Executive, 1(3), 207-219.

Tzafrir, S. S., \& Eitam-Meilik, M. (2005). The impact of downsizing on trust and employee practices in high tech firms: a longitudinal analysis. Journal of High Technology Management Research, 16(2), 193-207. doi: 10.1016/j.hitech.2005.10.008

Ulrich, D. (1997). Measuring human resources: an overview of practice and a prescription for results. Human Resource Management, 36(3), 303-320. doi: 10.1002/(SICI)1099050X(199723)36:3<303::AID-HRM3>3.0.CO;2-

Wall, T. D., \& Wood, S. J. (2005). The romance of human resource management and business performance, and the case for big science. Human Relations, 58(4), 429-462. doi: $10.1177 / 0018726705055032$

Way, S. A. (2002). High performance work systems and intermediate indicators of firm performance within the US small business sector. Journal of Management, 28(6), 765-785. doi: $10.1177 / 014920630202800604$

Way, S. A., \& Johnson, D. E. (2005). Theorizing about the impact of strategic human resource management. Human Resource Management Review, 15(1), 1-19. doi: 10.1016/j.hrmr.2005.01.004 
Wood, S. J. (1999a). Getting the measure of the transformed high-performance organization. British Journal of Industrial Relations, 37(3), 391-417. doi: 10.1111/1467-8543.00134

Wood, S. J. (1999b). Human resource management and performance. International Journal of Management Reviews, 1(4), 367-413. doi: 10.1111/1468-2370.00020

Wright, P. M., Gardner, T. M., Moynihan, L. M., \& Allen, M. R. (2005). The relationship between HR practices and firm performance: examining causal order. Personnel Psychology, 58(2), 409-446. doi: $10.1111 /$ j.1744-6570.2005.00487.x

Wright, P. M., \& McMahan, G. C. (1992). Theoretical perspectives for strategic human resource management. Journal of Management, 18(2), 295-320. doi: 10.1177/014920639201800205

Wright, P. M., \& Nishii, L. H. (2006). Strategic HRM and organizational behavior: integrating multiple levels of analysis [Working Paper $\mathrm{N}^{\circ}$ 405]. Cornell University, Ithaca, NY, USA.

Youndt, M. A., Snell, S. A., Dean, J. W., Jr., \& Lepak, D. P. (1996). Human resource management, manufacturing strategy, and firm performance. Academy of Management Journal, 39(4), 836866. doi: $10.2307 / 256714$

Zacharatos, A., Barling, J., \& Iverson, R. D. (2005). High-performance work systems and occupational safety. Journal of Applied Psychology, 90(1), 77-93. doi: 10.1037/00219010.90.1.77

Zhu, W., Chew, I. K. H., \& Spangler, W. D. (2005). CEO transformational leadership and organizational outcomes: the mediating role of human-capital-enhancing human resource management. Leadership Quarterly, 16(1), 39-52. doi: 10.1016/j.leaqua.2004.06.001 